\title{
Robot-assisted Optical Ultrasound Scanning
}

\author{
George Dwyer, Erwin J Alles, Richard J Colchester, Keshav Iyengar, Adrien E Desjardins, \\ and Danail Stoyanov, Senior Member, IEEE
}

\begin{abstract}
Optical ultrasound, where ultrasound is both generated and received using light, can be integrated in very small diameter instruments making it ideally suited to minimally invasive interventions. One-dimensional information can be obtained using a single pair of optical fibres comprising of a source and detector but this can be difficult to interpret clinically. In this paper, we present a robotic-assisted scanning solution where a concentric tube robot manipulates an optical ultrasound probe along a consistent trajectory. A torque coil is utilised as a buffer between the curved nitinol tube and the probe to prevent torsion on the probe and maintain the axial orientation of the probe while the tube is rotating. The design and control of the scanning mechanism are presented along with the integration of the mechanism with a fibre-based imaging probe. Trajectory repeatability is assessed using electromagnetic tracking and a technique to calibrate the transformation between imaging and robot coordinates using a known model is presented. Finally, we show example images of 3D printed phantoms generated by collecting multiple OpUS A-scans within the same 3D scene to illustrate how robot-assisted scanning can expand the field of view.
\end{abstract}

Index Terms-Medical Robotics, Robot-assisted Imaging, Optical Ultrasound

\section{INTRODUCTION}

O PTICAL Ultrasound (OpUS) is an emerging imaging modality where ultrasound is both generated and detected optically. The use of optical fibres has allowed for OpUS imaging probes to have exceptionally small diameters $(<1.0 \mathrm{~mm})$ [1], [2]. For a single pair of optical fibres (transmitter and receiver), an A-scan can be formed comprised of depth information from ultrasound reflections along a single axis. OpUS, as an interventional imaging modality, has a number of potential advantages over conventional piezoelectric based ultrasound. The small diameter of optical fibres means that OpUS is naturally suited to minimally invasive interventions; it is also electromagnetically compatible and the fibres can be produced at a relatively low cost.

There is promising potential for OpUS to be used as an alternative to conventional ultrasound in endoluminal procedures where flexible instrumentation is needed, for example

The work was supported by the Wellcome/EPSRC Centre for Interventional and Surgical Sciences (WEISS) [203145Z/16/Z]; an Innovative Engineering for Health award by Wellcome Trust [WT101957]; Engineering and Physical Sciences Research Council (EPSRC) [NS/A000027/1], the EPSRC-funded UCL Centre for Doctoral Training in Medical Imaging (EP/L016478/1), the EPSRC Healthcare Technologies Challenge Award [EP/N021177/1], EPSRC grants (EP/N027078/1, EP/P012841/1, EP/P027938/1, EP/R004080/1, EP/T517793/1), the Royal Academy of Engineering under the Research Fellowship Scheme; the Royal Academy of Engineering Chair in Emerging Technologies Scheme; and Horizon 2020 FET (GA 863146). For the purpose of Open Access, the author has applied a CC BY public copyright licence to any Author Accepted Manuscript version arising from this submission.

The authors are with Wellcome / EPSRC Centre for Interventional and Surgical Sciences, University College London (UCL), London, UK in endovascular surgery or cardiology [3], [4]. In minimally invasive surgery through a trocar port, OpUS can also be used, for example in fetal surgery where the amniotic fluid acts as an effective ultrasound medium and hence the imaging probe does not require direct contact with the interrogated tissue [5]. Fetal procedures, such as laser fetal surgery for twin-twin transfusion syndrome or fetoscopic endoluminal tracheal occlusion, would greatly benefit from small profile $(<5 \mathrm{~mm})$, forward looking imaging to support the identification of critical structures and blood vessels. Additional clinical applications could be in transurethral bladder investigations or resection, arthroscopic procedures or in water-assisted colonoscopy, but all would require larger field of view information than an A-scan. Here, we address this problem through robotics, and present the mechanical characterisation of a novel concentric tube robot aimed at versatile volumetric OpUS imaging. However, it is important to note that many important requirements for clinical use of OpUS and such robotic instrumentation would require extensive translational engineering and in vivo studies to determine imaging resolution, depth penetration, robotic motion speeds, biocompatibility and safety.

In the absence of scanning or rotation, OpUS probes with one transmitter/receiver pair yield only one-dimensional Ascans. One-dimensional structural information is inherently difficult to interpret as clinical information, for instance, when attempting to identify vasculature on and below the tissue surface. However, this information can be used to assist other modalities. For example, a distance-to-surface metric used as feedback for a controller of a robotic manipulator can be obtained with the use of the A-scan [5], [6]. This has been demonstrated in an instrument with a camera and a therapeutic laser, where the distance constraint provides improved white light camera images and improves performance in laser coagulation procedures. Alternatively, the receiver can be embedded into a needle, and used with a conventional ultrasound probe to assist in needle tracking [7]. Here only a Fabry-Pérot ultrasound receiver is integrated into the needle and is used to detect encoded transmissions from a modified transabdominal ultrasound transducer. This arrangement incorporates two arrays of unfocused transducers either side of the main imaging transducers; the arrival time of the signal detected by the embedded receivers from the arrays can be used to determine the $3 \mathrm{D}$ position of the needle relative to the ultrasound transducer. One approach to multi-dimensional information is to use manual scanning, where the operator moves the probe to build up a succession of A-lines over time, i.e. an M-mode image. This has been demonstrated in vivo with an OpUS integrated into a needle to guide a transseptal puncture procedure [4]. Here, the OpUS image provide both depth and tissue thickness feedback to guide the procedure. 
However, probe manipulation and image interpretation relies heavily on the operators experience.

However, to acquire structural information similar to conventional ultrasound, information is needed across multiple axes. A method of achieving this is using a galvano mirror and a cylindrical lens above a planar optical ultrasound generating membrane [8]. The mirrors scan excitation light across the membrane to generate ultrasound pulses from various spatial positions, which are detected by a single receiver, thus acquiring B-scans (two dimensional data, resembling traditional raw ultrasound data) at 15 frames per second. Similarly, fixed arrays of transducers can be fabricated using micro-machining techniques allowing them to fit a small form factor $(2.0 \mathrm{~mm})$ whilst being capable of volumetric imaging [9], [10]. For radial images, the transmitter fibre can be polished to $45^{\circ}$, coated with a mirror finish and rotated along the fibre axis to acquire a radial B-scan (similar to those acquired with intravascular ultrasound probes) [3]. Through the use of a robot manipulator, and scanning the probe across the target, a B-scan or C-scan (volumetric data) can be obtained as presented [11], [12]. However, this approach increases the size of the instrument and introduces large acquisition times when obtaining the imaging information.

Dedicated robotic scanning mechanisms can be utilised to assist in imaging by manipulating the imaging probes in a known and repeatable manner. This approach has been applied to other modalities which suffer from limited fields of view or provide only one dimensional information. For example, endomicroscopy is often the targeted modality for scanning mechanisms as it has a high resolution but limited field of view (for confocal endomicroscopy, approximately $2 \mu \mathrm{m}$ and $250 \mu \mathrm{m}$ respectively) which limits the clinical relevance. Endomicroscopy requires tissue contact and device orientation approximately perpendicular to the tissue surface, therefore the scanning mechanisms often focus on providing two translational degrees of freedom to manipulate the probe in a known and repeatable pattern and then combining the recorded images. To counter trajectory errors introduced from tissue deformation (as the probe must be in direct contact with the tissue), a visual servoing approach is introduced to adjust the trajectory while scanning. Images are then combined using visual information but each image is initialised in the estimated kinematic position. Small diameter (approximately $2 \mathrm{~mm}$ ) high resolution images have been presented using scanning mechanisms to assess surgical margins in tumour resection procedures [13]-[15]. Alternatively, scanning mechanisms to generate larger mosaics to compare with ultrasound have been proposed to combine information from different axes [16]. A further example is a scanning fibre endoscope where 1D information is utilised to develop a 2D image. A single fibre with a collimating lens is coupled to a white light source and scanned across a spiral pattern using a piezo tube actuator; the reflected light is detected by a static sensor and used to construct a 2D image [17]. Lastly, 1D modalities such as single array ultrasound or photoacoustic imaging applied to intraluminal imaging can utilise scanning mechanisms to acquire 3D information. By rotating and translating along the axis of the lumen allows radial images to be acquired and then stacked together to form a 3D volume [18]. Dedicated scanning mechanisms that are optimised for an imaging modalities characteristic have the potential to improve the clinical utility of the information acquired. A scanning mechanism targeted toward OpUS could allow for two- and three-dimensional imaging whilst maintaining a low profile to minimise the invasiveness of the instrument.

In this paper, we present the use of a concentric tube manipulator as a scanning mechanism for OpUS imaging probes. The scanning mechanism relies on repeatedly rotating a curved nitinol tube with an imaging probe about the instrument axis, whilst also extending and retracting the curved tube from the instrument shaft. This scanning mechanism allows for the synthesis of much larger imaging apertures than can be achieved using rigid probes under the size constraints of minimally invasive surgery, as well as for arbitrary spatial sampling density. In the remainder of this manuscript, the design of the 2 DoF mechanism is presented in detail, the repeatability of the mechanism is assessed and, finally, the mechanism is demonstrated through the provision of C-scans using an OpUS sensor in a laboratory phantom model environment.

\section{INSTRUMENT DESIGN}

One of the primary advantages of OpUS is the ability to integrate imaging capabilities within very small profile instrumentation like catheters used in endovascular and cardiovascular interventions where the instrument moves within blood vessels. In this paper, we consider the potential application of OpUS in endoscopic or laparoscopic approaches, where the instruments are typically operated under white light illumination and imaging, observing a forward looking, wide field of view of the surgical site.

The purpose of the OpUS scanning mechanism that we have designed is to manipulate the forward viewing OpUS probe across a consistent trajectory, while continuously acquiring OpUS data. Volumetric images can then be generated from the kinematic data yielding the actuator pose for each acquired A-scan. A concentric tube mechanism consisting of a straight and rigid outer tube combined with a rotating and curved elastic inner tube arranged concentrically is used to manipulate the probe. The rotation and translation of the inner tube is actuated, allowing the inner tube to be manipulated relative to the outer tube. The inner tube is actuated on a moving carriage using a lead screw for the linear motion, and on a rotating shaft with a sliding fit for the rotation. This approach allows the motors to be fixed in position at the proximal end of the instrument. Through manipulation of its extension, the deflection angle of the inner tube can be controlled. Similarly through rotation, the deflection plane can be orientated about the instrument axis. When a highly directional OpUS imaging probe is introduced into the inner tube, the actuator mechanism allows for two-dimensional control over the orientation of an emitted ultrasound beam - thus enabling the synthesis of imaging apertures with spatial dimensions that far exceed the physical footprint of the device, which can be scanned at arbitrary spatial sampling density. 

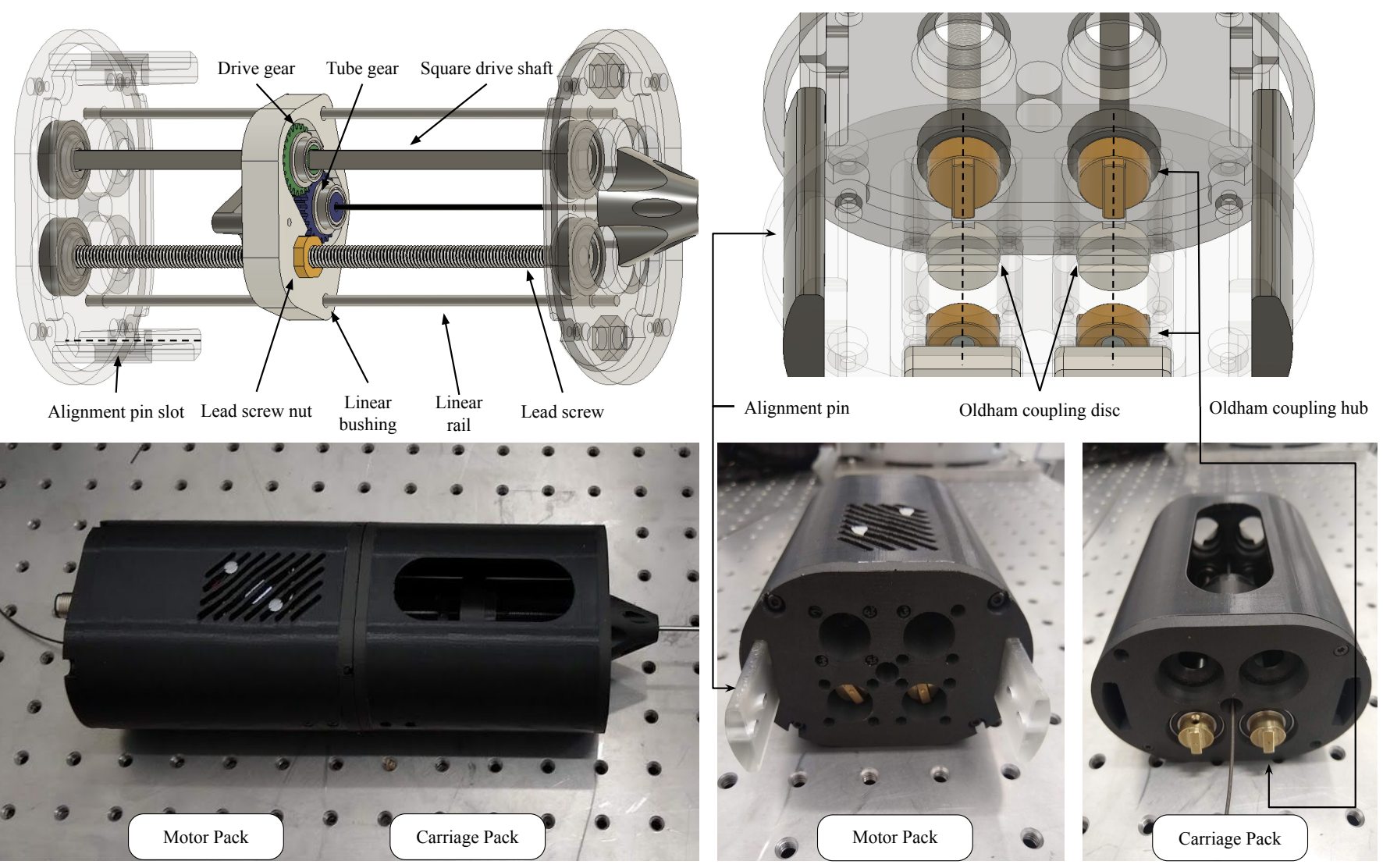

Fig. 1. Top Left - CAD rendering of the carriage pack, illustrating the shaft assemblies running the length of the pack constrained by bearings at either end and an Oldham hub to couple with the motor pack. The carriage is supported in the centre of the housing by the shaft assemblies and the cylindrical rails running across the centre of the housing. Top Right - CAD rendering of the coupling between the motor pack and carriage pack. Black dotted line shows the alignment between the motor Oldham hub, the Oldham disc and the carriage Oldham hub with the alignment pins on either side fixed to the motor pack. Bottom Row - Images of the assembled scanning mechanism: Left showing the coupled carriage and motor section, Centre showing the motor section with the coupling pins, Right showing the carriage section.

\section{A. Mechanism Design}

The proposed instrument has a modular design consisting of two main sections: motor pack and carriage pack. The motor pack has the capacity for four actuators, allowing the control of two tubes for more complex instrumentation. However, for this application, control of only a single tube was required. The actuators to drive the mechanism were arranged about the central axis of the tube. Stepper motors were selected due to their high repeatability and the ability to apply relatively high torque at low speeds. The motor pack is joined to the carriage pack through two alignment pins and the motion of the actuators is transferred through Oldham couplings. Fig. 1 illustrates the carriage pack with the carriage and shaft assemblies within, along with the assembled fabricated instrument. Oldham couplings were selected for three main features. First, Oldham couplings can transmit torque with virtually no backlash, a key source of joint error. Second, the disc in between the hubs operates as a torque limit. Once a disc breaks due to torque overload, both shafts can rotate independently. Last, a sterile drape can be placed between the motor pack and carriage back with minor modifications, allowing the instrument to be sterilised [19]. Oldham couplings consist of three components: two hubs, typically metal, that couple to the input and output shafts and have a single rail across the face of the hub; a disc, constructed typically from a plastic with low friction. The disc has a slot for the rail on each face and the slots are offset $90^{\circ}$ from each other. This arrangement allows for some translational error in the positioning of the shafts as the disc will slide along the rails to compensate. Fig. 1 shows the coupling between the motor pack and carriage pack where the alignment pins are extended beyond the Oldham couplings, a design feature incorporated to ensure alignment before the couplings are engaged. The alignment pins are then fixed with a M2.5 cap screw to prevent the packs from decoupling unintentionally.

The main components of the carriage pack include the the carriage, lead screw assembly and square shaft assembly. The carriage holds the drive gear (shown green in Fig. 1), with the square shaft running through it and allowing the gear to traverse along its length and rotate if the square shaft is driven. The drive gear is meshed with the tube gear (shown blue in Fig. 1) that holds the curved nitinol tube, while the lead screw nut is constrained within the carriage housing and uses the two linear rails and square shaft to act as the runner when the lead screw is engaged. Linear bushings (GSM-0304-03, igus $\mathrm{GmbH}, \mathrm{DE}$ ) provide the interface between the carriage and the linear rails and are friction-fit on the sides of the carriage. Each shaft assembly consists of the lead screw or square shaft 
held by a small coupling component that constrains the shaft to the centre of a cylindrical outer profile. Radial bearings hold the coupling component to the housing at each end. The coupling at the proximal side of the carriage section has a $4 \mathrm{~mm}$ pin to fix the Oldham coupling and on the opposing side, the shaft profile with grub screw fixings placed radially about the profile. The distal side coupling component has the shaft profile throughout to prevent length alignment issues between the other shaft assembly and the housing. The shaft assemblies are held within the carriage housing which has separate front and back plates that hold the shaft assembly bearings and a uniform housing profile. A significant advantage of this arrangement is that it allows for the length of the carriage pack, and therefore the linear joint range of the mechanism, to be easily modified.

The majority of the components were fabricated using a 3D printer (Mark Two, Markforged Inc., US) in Onyx, with the exception of the central housing of the motor pack and carriage pack, the coupling pins, and the drive and tube gears in the carriage. Onyx is nylon, mixed with chopped carbon fibre to strengthen the material and this was used for the main components due to the printer's comparatively high dimensional accuracy. The material is significantly stronger than Polylactic Acid (PLA) and provides heat deflection at a temperature of $145^{\circ} \mathrm{C}$. This property is required as the heat deflection temperature of PLA is generally below $60^{\circ} \mathrm{C}$ and the temperature of stepper motors can readily exceed $60^{\circ} \mathrm{C}$. The use of Onyx prevents the deformation of the motor pack whilst under load. The tube and drive gears are made in Iglidur I6PL (igus $\mathrm{GmbH}, \mathrm{DE}$ ), using a selective laser sintering printer. This material was chosen as it has high abrasion resistance and good sliding properties. Both gears have the same modulus and pitch circle diameter of 0.75 and $18 \mathrm{~mm}$ respectively giving a 1:1 gear ratio. The main housing sections are printed from PLA using an Ultimaker S5 (Ultimaker B.V., NL), as the load applied to them is low and they do not need the heat resistance properties of Onyx. The coupling pins were printed using a Form 2 (Formlabs Inc., US) with clear resin to provide a rigid and smooth finish to ease coupling. The square shaft is stainless steel $5 \mathrm{~mm}$ square bar stock, and the lead screw is stainless steel with a $6 \mathrm{~mm}$ diameter and $1 \mathrm{~mm}$ lead. The lead screw nut is made from Iglidur $\mathbf{J}$ (igus $\mathrm{GmbH}, \mathrm{DE}$ ) and has a filleted square profile that allows it to slot into the housing with a friction fit with no need for an additional radial locking mechanism.

The stepper motors are NEMA 8 (ST2018, Nanotec Electronic $\mathrm{GmbH} \& \mathrm{Co} . \mathrm{KG}, \mathrm{DE})$, and these can provide $3.6 \mathrm{Ncm}$ of holding torque and operate in steps of $1.8^{\circ}$. The motors can apply a torque of $2.8 \mathrm{Ncm}$ at $10 \mathrm{rev} / \mathrm{s}$, which for the linear joint translates to $10 \mathrm{~mm} / \mathrm{s}$ and a resolution of $5 \mu \mathrm{m}$. The motors have a frame size of $20 \times 20 \mathrm{~mm}$ and $48 \mathrm{~mm}$ length. These are positioned in the motor pack with slotted vents along the side and a small fan to cool the motors while in operation. The final assembled instrument, shown in Fig. 1, has a total length of $235 \mathrm{~mm}$, consisting of $125 \mathrm{~mm}$ and $110 \mathrm{~mm}$ for the motor section and carriage section respectively.

\section{B. Optical Ultrasound Sensor and System}

The OpUS sensor consists of a fibre-optic ultrasound transmitter and a fibre-optic ultrasound receiver. The ultrasound transmitter comprised a multi-walled carbon nanotube (MWCNT) and polydimethylsiloxane (PDMS) composite [1], [2] coated onto the distal end surface of a $400 \mu \mathrm{m}$ core multimode optical fibre. The ultrasound receiver comprised a planoconcave Fabry-Pérot cavity fabricated on the distal end surface of a single mode optical fibre (SMF-28) [20]. The two optical fibres were held adjacent, and heat shrink tubing was used to align their distal end surfaces. The probe was interrogated using a system previous described [3]. This type of OpUS probe has previously been extensively characterised [4], [21], and emitted a pressure of $8.8 \mathrm{MPa}$ at a distance of $1.5 \mathrm{~mm}$, at a wide bandwidth of $26.5 \mathrm{MHz}$ (centered around $20 \mathrm{MHz}$ ). Through the use of a large (relative to the acoustic wavelength) source element, ultrasound is emitted in highly directional fashion: a divergence angle of $15.2^{\circ}$ was found when the bandwidth was confined to $20-40 \mathrm{MHz}$, resulting in a lateral resolution of $1.6 \mathrm{~mm}$ at an axial depth of $5 \mathrm{~mm}$.

\section{OpUS Instrument}

In order to integrate the OpUS probe into the concentric tube mechanism a number of mechanical challenges need to be addressed. Firstly, the OpUS probe must retain its original axial orientation because continuous twisting would snap the fibres. Secondly, the fibres need to remain at the tip of the curved inner tube while the tube is translated. To accommodate the design constraints outlined above, a torque coil (Nitinol Helical Hollow Strand tubing, Fort Wayne Metals Ireland Ltd, IE) was integrated into the design. The main advantage of torque coil is that while highly flexible and compliant, it exhibits high torsional rigidity. Torque coils are often utilised to apply torque through catheter like devices that also require lateral flexibility such as milling tools for orthopaedic surgery [22] or for rotational scanning mechanisms to get radial images [18]. The torque coil has a outer diameter and inner diameter of $1.26 \mathrm{~mm}$ and $0.88 \mathrm{~mm}$ and consists of 8 strands of Nitinol formed into a single layer helical strand. The optical ultrasound probe is held within the torque coil, which in turn is within the curved nitinol tube. The torque coil is fixed in position between the linear and rotational joints of the curved nitinol tube. This moves the torque coil linearly in unison with the curved nitinol tube, but fixes the orientation of the tip while the curved nitinol tube rotates. The torque coil thus effectively acts as a sheath protecting the optical ultrasound probe against twisting and friction. The assembled instrument consists of a stainless steel instrument shaft rigidly fixed to the carriage pack, $185 \mathrm{~mm}$ long, with an outer and inner diameter of $2.45 \mathrm{~mm}$ and $1.8 \mathrm{~mm}$ respectively. Within the instrument shaft is the curved nitinol tube coupled with the carriage in the carriage pack with an outer and inner diameter of $1.59 \mathrm{~mm}$ and $1.4 \mathrm{~mm}$ respectively. The nitinol tube was shape-set to an arc with a bending radius of $35 \mathrm{~mm}$ and an arc length of $40 \mathrm{~mm}$. The OpUS probe within the torque coil is then positioned within the nitinol tube at the tip and fixed in position at the 

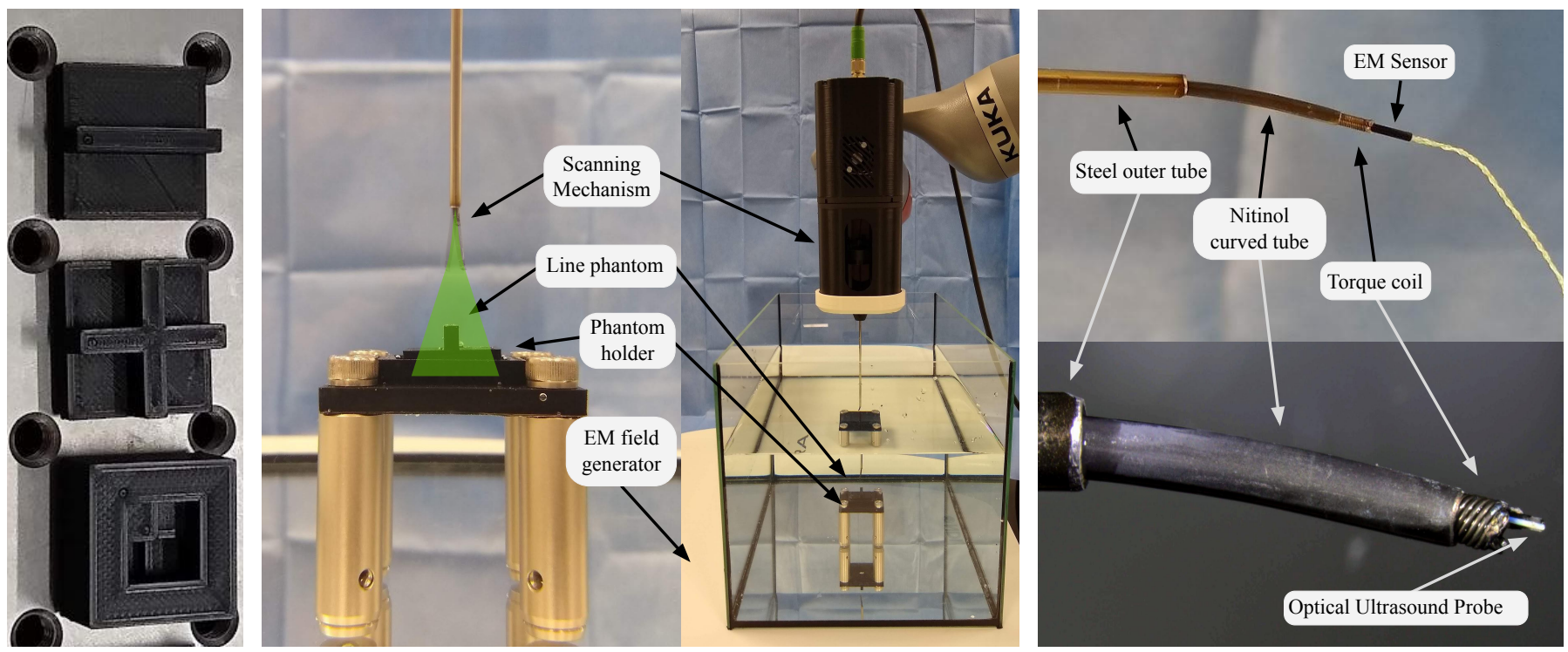

Fig. 2. Left - the range of printed imaging phantoms used to assess the quality of the C-scans. From top to bottom: line phantom, cross phantom, and stepped phantom. Centre - the experimental setup for acquiring scans of phantoms: with the phantom held in the phantom holder and the scanning mechanism positioned above (ca. $20 \mathrm{~mm}$ ) and the centre of the phantom with the instrument axis normal to the phantom. Green overlay illustrates the approximate field of view of the scanning mechanism. Right - the two configurations of the scanning mechanism used, Top - with the EM sensor inserted into the torque coil from the distal end, Bottom - with the OpUS sensor extruded from the tip of the torque coil.

carriage. The sensor assembly at the distal end of the scanning mechanism is shown in Fig. 2.

\section{Mechanism Control}

Each axis of the mechanism is controlled using a dedicated controller (CL3-E-1-0F, Nanotec Electronic GmbH \& Co. KG, $\mathrm{DE}$ ) and powered from a $12 \mathrm{~V}$ power supply. Communication to the controller is through the Controller Area Network (CAN) protocol (a multi-master message-based communication protocol). Each controller allows the motor to run in open loop mode but with the rotation motor running in velocity mode and the linear motor running in profile position mode.

The arrangement, as described above, provides the control in actuator space but the mechanism information needs to be accounted for in order to move in joint space. The mapping between actuator values and joint values can be shown as $q=k(A+\lambda)$, where $q$ is the joint state, $k$ is the gear ratio, $A$ is the actuator state and $\lambda$ is the zero offset. The joint state is composed of the rotation of the tube and extension of the inner curved tube from the outer tube (steel instrument shaft). The actuator state is the angle of the actuator and the zero offset is the actuator angle at the joint zero position. The gear ratio for the rotation joint is the gear ratio between the drive and tube gears within the carriage, and for the linear joints the gear ratio is the lead of the lead screw.

For the assembled mechanism, this resulted in a gear ratio of 1.0 and 0.001 for the rotation and linear joint respectively. A forward kinematics solver with backlash compensation was implemented using the domination stiffness method [23]. The compensation is used to account for free motion from mechanical tolerances or elastic effects when a joint is changing direction. A backlash threshold is used to compare current and previous joint positions to determine if the direction has changed and compensation is required. If necessary, the compensated position remains unaltered until the difference in position has exceeded the backlash value. The compensated position is then used to calculate the tube transform with the domination stiffness method.

\section{E. Optical Ultrasound Processing}

The processing of the optical ultrasound using the kinematics of a manipulator was previously presented in [11], with the signal processing presented in detail in [3], [24]. Briefly, during operation, the unprocessed A-scans and kinematic information are recorded with timestamps using rosbags. The completed scans are then post-processed in Matlab using the following procedure: The kinematic information is synchronised with the unprocessed A-scans using the timestamps; as the A-scans are published at approximately $100 \mathrm{~Hz}$ while the joint states are published at $500 \mathrm{~Hz}$; the joint states are compared to the A-scans and the joint state closest in time to each A-scan is found. Following the synchronisation, the A-scans are processed through frequency filtering, envelope detection and log-compression. The processed A-scans are then transformed using the matched kinematic pose from the sensor frame to a common coordinate frame which, in this instance, is the tip of the outer tube. Each resulting A-scan is formed as a point cloud and sequentially added together to form a C-scan. After the addition of an A-scan, a $10 \mu \mathrm{m}$ voxel filter is applied to the $\mathrm{C}$-scan preventing large sections of spatially overlapping data. Finally, an empirically determined intensity threshold was applied to the constructed C-scan to optimise the display.

\section{F. Scanning Mechanism Calibration}

With the scanning mechanism and controller assembled and operational, a number of unknown parameters remain 
that complicate accurate imaging. The first parameter to be determined is the curvature of the nitinol tube with the OpUS sensor and torque coil integrated. This requires identifying the kinematic parameters of the concentric tube robot. The second is the free axial motion or backlash between the OpUS sensor and the linear joint. The backlash of the rotation joint is not a consideration because the direction of rotation is constant. This free axial motion is a combination of actual backlash from the lead screw driving the carriage and elastic effects from the curved nitinol tube and torque coil.

To estimate these parameters, the scanning mechanism is used to image a phantom with known geometric properties: in this case, a planar surface with a line $3 \mathrm{~mm}$ wide and $5 \mathrm{~mm}$ high through the centre (3D printed using an Ultimaker S5) shown in Fig. 2. The calibration process is as follows. First the scanning mechanism is positioned in a static position above the phantom, the mechanism is activated and the OpUS information and kinematic data are recorded. C-scans are then generated across a range of backlash and tube curvature values following the procedure presented in the previous subsection (§II-E). Each C-scan is then passed through a threshold filter to remove the low intensity points. This isolates the two top faces of the phantom: the raised line and the base. With these two faces, two metrics are then calculated; the distance between a fitted plane at each face, the width of the raised line. Subsequently, the calibrated parameters are found from the minimised error between the $\mathrm{C}$-scan and the measured dimensions of the raised calibration line.

\section{EXPERIMENTS AND RESULTS}

\section{A. Experimental Setup}

Fig. 2 shows the experimental setup for acquiring the scan data using the scanning mechanism. The scanning mechanism is held over a tank of water with the instrument shaft submerged and a phantom holder at the base of the tank. The phantom holder raises the phantom from the base of the water and fixes to the phantom using an M6 screw. Four phantoms are used each with a $20 \mathrm{~mm} \times 20 \mathrm{~mm}$ base with a plane, raised line, raised cross or multiple stepped levels as surface features. The raised line and cross have a $3 \mathrm{~mm}$ wide, $5 \mathrm{~mm}$ high line running along a single axis and two axis, respectively. The stepped phantom has a $5 \mathrm{~mm}$ border around the outside of the phantom; in the centre a grid layout with four levels recessed at 2,4,6,8 $\mathrm{mm}$ offset from the border and each has an area of $5 \mathrm{~mm} \times 5 \mathrm{~mm}$. The plane phantom was a single sheet of acrylic $2 \mathrm{~mm}$ thick while the remainder of the phantoms were printed using an Ultimaker S5 using a $0.25 \mathrm{~mm}$ nozzle and PLA with $20 \%$ infill and $0.1 \mathrm{~mm}$ layer height. The phantoms were measured with digital calipers (Axminster Tool Centre Ltd, UK), with a resolution of $0.01 \mathrm{~mm}$, to determine the physical dimensions. The cross and line phantoms had a measured width of $3.11 \mathrm{~mm}$ and a height of $5.02 \mathrm{~mm}$. For the stepped phantom the measured heights from the border were $2.02 \mathrm{~mm}, 4.00 \mathrm{~mm}, 6.01 \mathrm{~mm}, 8.02 \mathrm{~mm}$. Figure 2 shows the $3 \mathrm{D}$ printed phantoms designed to assess the C-scans.

The mechanism is positioned so the rigid instrument shaft (outer tube) is orientated approximately normal to the phantom

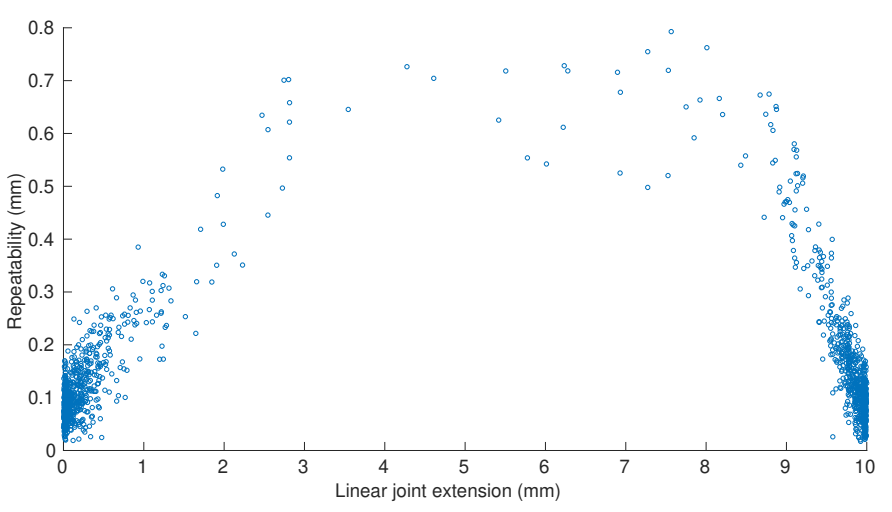

Fig. 3. The repeatability measurements of the scanning mechanism, using 7632 transforms clustered into 1465 groups. The measurements are displayed against the extension of the linear joint, with an average repeatability of $145 \mu \mathrm{m}$, and a maximum and minimum of $793 \mu \mathrm{m}$ and $17 \mu \mathrm{m}$ respectively.

surface, centred on the phantom and raised $20 \mathrm{~mm}$ above it. Scans are acquired with the mechanism running at $2 \mathrm{rev} / \mathrm{s}$ on the rotation joint and, for the linear joint, at between $0.0 \mathrm{~mm}$ and $10.0 \mathrm{~mm}$ with a maximum velocity of $10 \mathrm{~mm} / \mathrm{s}$ and a maximum acceleration is $5 \mathrm{~mm} / \mathrm{s}^{2}$.

\section{B. Scanning Mechanism Trajectory Assessment}

As the mechanism is designed to consistently follow the same spiral trajectory, the repeatability of the motion needs to be assessed. To enable this, the OpUS probe is removed from the torque coil and replaced with an EM tracking sensor (Aurora, NDI Inc., CA). The sensor can be tracked with 5 DoF and is $11 \mathrm{~mm}$ long with a diameter of $0.8 \mathrm{~mm}$, allowing it to be inserted into the torque coil at the tip. A tabletop field generator (Aurora, NDI Inc., CA) is placed below the instrument, the tracking system has a RMS accuracy of $1.2 \mathrm{~mm}$ and precision and trueness of $0.7 \mathrm{~mm}$ and $1.0 \mathrm{~mm}$. Fig. 2 shows the sensor and the overlapping section of torque coil extended from the nitinol tube. This is necessary to prevent deformation of the sensor from the interactions between the nitinol inner tube and steel outer tube.

With the sensor attached at the tip of the mechanism a repeatable scanning trajectory was used. This trajectory is set to travel between $0.0 \mathrm{~mm}$ and $10.0 \mathrm{~mm}$ with the linear joint and at a constant velocity of $2 \mathrm{rev} / \mathrm{s}$ in rotation. The linear joint is set with a trapezoidal velocity profile with a maximum velocity of $10 \mathrm{~mm} / \mathrm{s}$ and maximum acceleration of $5 \mathrm{~mm} / \mathrm{s}^{2}$. The scanning mechanism is set to follow this trajectory for 20 minutes and the joint states and tracker transforms are timestamped to the system timer and recorded. Once completed, the tracker positions are aligned with the joint states through the timestamps (the tracker outputs at approximately $40 \mathrm{~Hz}$ while the mechanism controller outputs joint states at approximately $500 \mathrm{~Hz}$ ). The synchronisation procedure results in 48,021 aligned joint states and transforms. The joint states were sorted into bins of $0.2^{\circ}$ and $50 \mu \mathrm{m}$, resulting in 1465 unique groups with at least 4 entries. The respective transforms of the matched groups were then taken and the standard deviation across the groups was calculated, resulting in an average repeatability of $145 \mu \mathrm{m}$, and a maxi- 

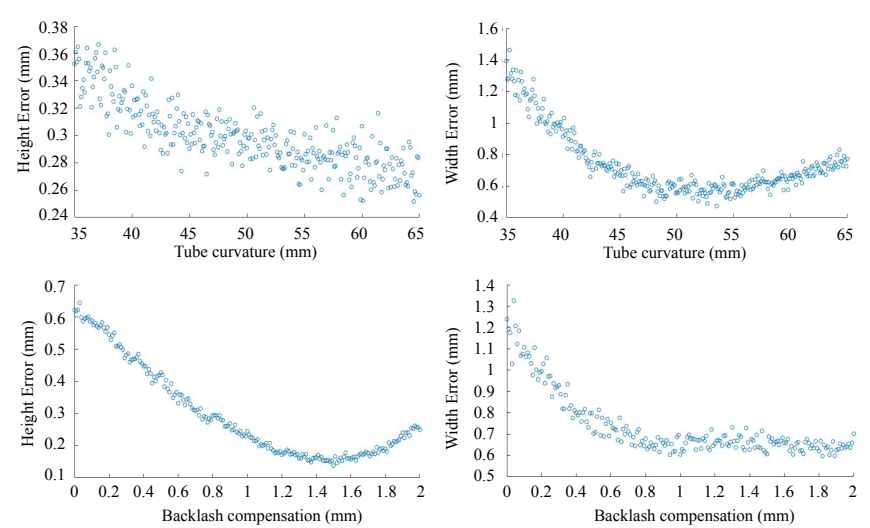

Fig. 4. The two error metrics of the line calibration: Left column - height error between two fitted planes of the line and the base of the phantom; Right column - the error between the width of the top surface of the C-scan and the width of the physical phantom top surface. For each column, Top shows the average fitting error against tube curvature, and Bottom shows the average fitting error against mechanism backlash.

mum and minimum of $793 \mu \mathrm{m}$ and $17 \mu \mathrm{m}$ respectively. Fig. 3 shows the measured repeatability over the linear range of the mechanism. Additionally, tracked poses from the EM tracker were recorded over the range of linear motion in a scan but at a fixed tube orientation. A sphere was fit to the tracked position to measure the bending radius of the curved tube: the fitted sphere had a radius of $49.5 \mathrm{~mm}$ with a mean error of the distance between the points and the model of $91 \mu \mathrm{m}$.

\section{Scanning Mechanism Calibration}

The line phantom is used to calibrate the scanning mechanism with the data acquired as described in section III-A. The calibrated parameters are then determined through a search over both the backlash and the tube curvature. The backlash range was from $0 \mathrm{~mm}$ to $2 \mathrm{~mm}$, in $10 \mu \mathrm{m}$ increments, while the tube curvature is from $35 \mathrm{~mm}$ to $65 \mathrm{~mm}$ in $500 \mu \mathrm{m}$ increments. Fig. 4 shows the height and width error with respect to the tube curvature and backlash separately. The set of parameters that resulted in the minimum error from the line phantom were $53.5 \mathrm{~mm}$ and $1.49 \mathrm{~mm}$ for the tube curvature and backlash, respectively. Fig. 5 shows the influence of the parameters on the C-scans. The uncalibrated C-scan parameters assume the curvature of the tube conforms to the curvature of the jig in which the nitinol was set $(35 \mathrm{~mm})$ and that the mechanism has no backlash.

\section{Reconstruction Assessment}

The quality of the reconstructions is then assessed through the use of a range of 3D printed phantoms. Fig. 6 shows the $\mathrm{C}$-scan of the plane phantom where the top and bottom surfaces of the plane are shown in blue and green, respectively. The centre and edges of the scan show an edge likely from axial motion of the OpUS that was not compensated for in the calibration procedure. The top surface of the plane is manually segmented from the bottom surface and is assessed in relation to two considerations: how planar is the surface, and what is the deviation from the mean surface height. A plane is fit to
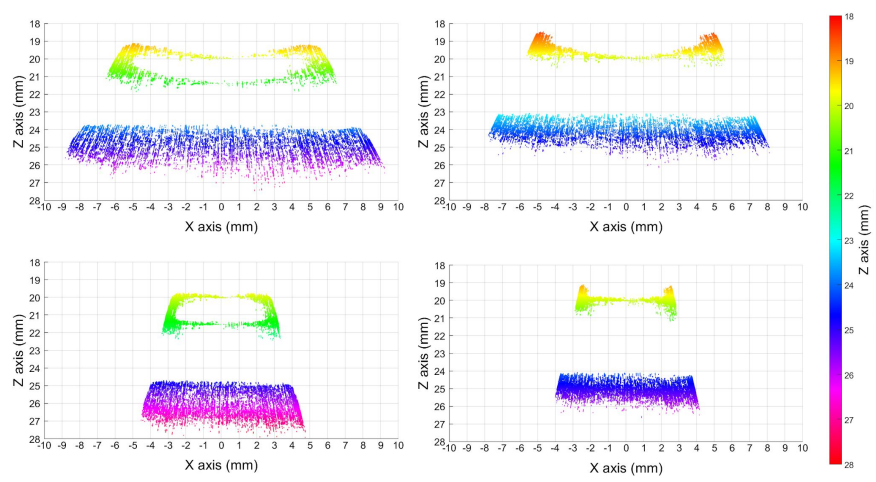

Fig. 5. C-scans of the line phantom shown constructed using different parameters, with the origin of the axis at the tip of the instrument shaft. The colour of the points is encoded to the depth along the $\mathrm{Z}$ axis. Top left - uncalibrated C-scan, Top right - backlash calibrated C-scan, Bottom left curvature calibrated C-scan, Bottom right - calibrated C-scan.
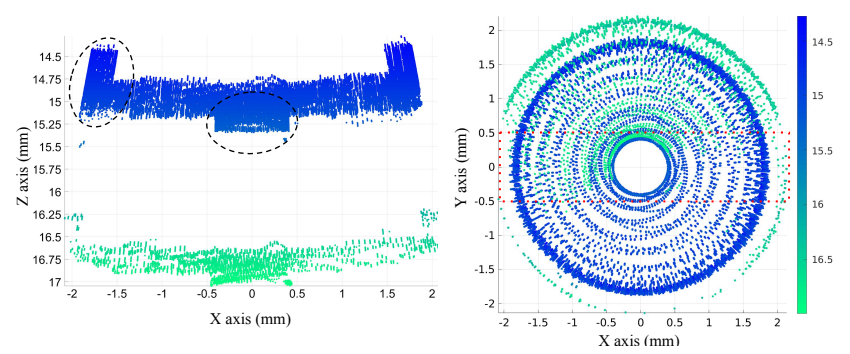

Fig. 6. The constructed C-scan of the plane phantom. The colour of the points is encoded to the depth along the $\mathrm{Z}$ axis. Left - side on sectioned view of the plane with the black dotted line providing an example of residual unmodelled axial motion. Right - top down view of the phantoms, with the red dotted line showing the subset displayed in the left figure.

the surface where the mean and median distance of the points to the plane were $0.19 \mathrm{~mm}$ and $0.14 \mathrm{~mm}$, with a maximum distance of $0.76 \mathrm{~mm}$. The standard deviation of all the points is $0.15 \mathrm{~mm}$.

Fig. 7 shows the C-scans of the line and cross phantoms. The line phantom is the same phantom used for the calibration but the image is the result of a different data set. Fig. 7 shows the line and cross phantoms in $\mathrm{XY}$ and $\mathrm{XZ}$ projections, with the depth of the points on the $\mathrm{Z}$ axis shown by the colour of the points. The left sub figure shows the motion of OpUS sensor to acquire the $\mathrm{C}$-scan with the $\mathrm{C}$-scan of the line phantom. For the line phantom, in addition to the surface assessment previously described for flatness and deviation from the mean of the surface; the width of the raised line is assessed along with the distance between the top and bottom planes using the measured geometry of the phantom. For the top plane, the mean and median distance of the points to the plane are $0.20 \mathrm{~mm}$ and $0.14 \mathrm{~mm}$ with a maximum distance of $0.99 \mathrm{~mm}$. The standard deviation of all the points was $0.19 \mathrm{~mm}$. For the bottom plane, the mean and median distance of the points to the plane are $0.31 \mathrm{~mm}$ and $0.25 \mathrm{~mm}$ with a maximum distance of $1.09 \mathrm{~mm}$. The standard deviation of all the points was $0.23 \mathrm{~mm}$. The distance between the fitted planes is $5.03 \mathrm{~mm}$. It was found that $98.36 \%$ of points lie within a width of $3.11 \mathrm{~mm}$ and $99.01 \%$ of points lie within a width of $3.66 \mathrm{~mm}$.

For the cross phantom, the top and bottom surfaces are 

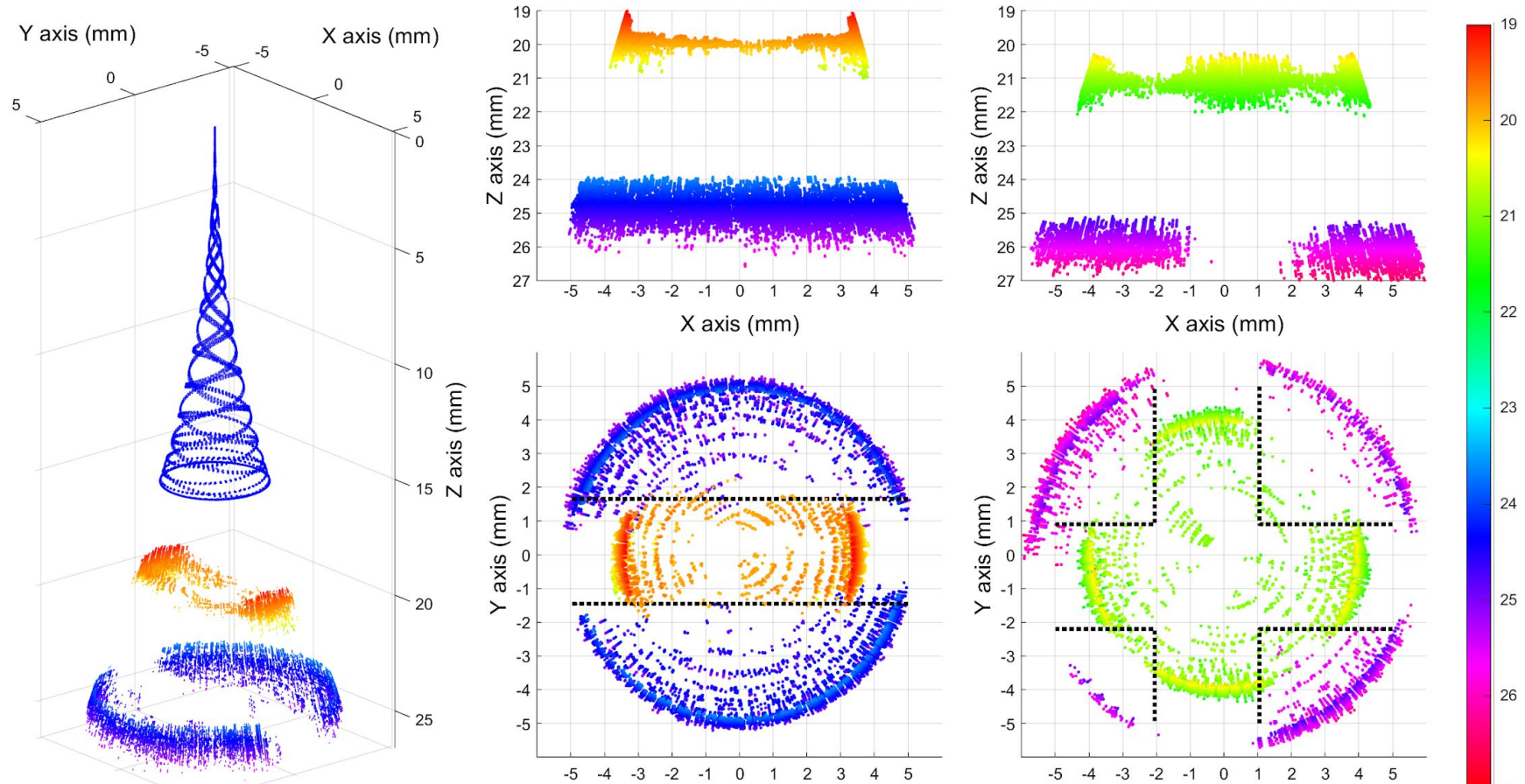

$\widehat{\xi}$
है
$\frac{n}{x}$
N
$N$

Fig. 7. The constructed C-scans of the line and cross phantoms. The colour of the points is encoded to the depth along the Z axis. Left - Angled view of the C-scan of the line phantom with the scanning trajectory of the OpUS probe shown in blue; Centre Column - line phantom; Right Column - cross phantom; Top row - side view of the phantoms. Bottom row - top down view of the phantoms, with the black dotted line showing the measured width of the line and cross phantoms, $3.11 \mathrm{~mm}$.

TABLE I

METRICS ON SCAN QUALITY FOR THE PLANE, LINE AND CROSS PHANTOMS

\begin{tabular}{cc|ccc} 
Phantom & & Plane & Line & Cross \\
\hline \hline \multirow{3}{*}{ Base plane deviation (mm) } & Mean & 0.19 & 0.31 & 0.31 \\
& Median & 0.14 & 0.25 & 0.28 \\
& Max & 0.76 & 1.09 & 0.94 \\
\hline \multirow{3}{*}{ Top plane deviation (mm) } & Mean & N/A & 0.2 & 0.26 \\
& Max & N/A & 0.14 & 0.21 \\
& N/A & 0.99 & 0.92 \\
\hline Distance between planes (mm) & N/A & 5.03 & 4.94 \\
Percentage of points within template & N/A & $98.36 \%$ & $95.90 \%$
\end{tabular}

assessed in the same way as the plane and additionally the width of each strut of the cross is assessed along with the distance between the top and bottom planes. For the top plane, the mean and median distance of the points to the plane are $0.26 \mathrm{~mm}$ and $0.21 \mathrm{~mm}$ with a maximum distance of $0.92 \mathrm{~mm}$. The standard deviation of all the points is $0.20 \mathrm{~mm}$. For the bottom plane, the mean and median distance of the points to the plane are $0.31 \mathrm{~mm}$ and $0.28 \mathrm{~mm}$ with a maximum distance of $0.94 \mathrm{~mm}$. The standard deviation of all the points is $0.22 \mathrm{~mm}$. The distance between the fitted planes is $4.94 \mathrm{~mm}$. The cross is found using a template of the cross which moves along the $\mathrm{X}$ and $\mathrm{Y}$ axis to find the maximum number of points within the template. Using the width of the printed cross, $3.11 \mathrm{~mm}, 95.9 \%$ of the points were within the template, while $99 \%$ of the points fit within a cross of $3.6 \mathrm{~mm}$. The results for the plane, line and cross are summarised in Table. I.

Fig. 8 shows the stepped phantom $\mathrm{C}$-scan and CAD renders coloured to the C-scan colourmap. For the stepped phantom, the levels of the phantom can be distinguished readily, as seen in the bottom-left section of Fig. 8. Five separated levels can be discerned: they are coloured as dark blue, light blue, green, yellow and red, with the top of each level separated by approximately $2 \mathrm{~mm}$ and fitting with the printed phantom. However, while the top two surfaces (red and green) and the bottom surface (purple) can be identified (the bottom surface being the base of the phantom), the two lower levels of the phantom are only slightly visible in a top-down view. The relatively large amount of noise in comparison to the previous phantoms did not allow the same level of analysis according to the geometry of the phantom.

\section{DISCUSSION}

This paper presents the utilisation of a concentric tube manipulator for robot-assisted scanning of an OpUS sensor that consists of a single transmitter and receiver pair acquiring one-dimensional information, an A-scan. The concentric tube manipulator allows the orientation of the sensor to be controlled about two axes while a torque coil between the OpUS sensor and the curved nitinol tube maintains the axial orientation of the sensor during scanning. A C-scan of the inspected area can be constructed by utilising the kinematics of the mechanism to determine the pose of the sensor for each A-scan. The overall instrument diameter is $2.45 \mathrm{~mm}$, and with the current scanning mechanism configuration (tube curvature and scanning trajectory), the maximum offset radially from the instrument axis during scanning is less than $3.0 \mathrm{~mm}$. Both the 


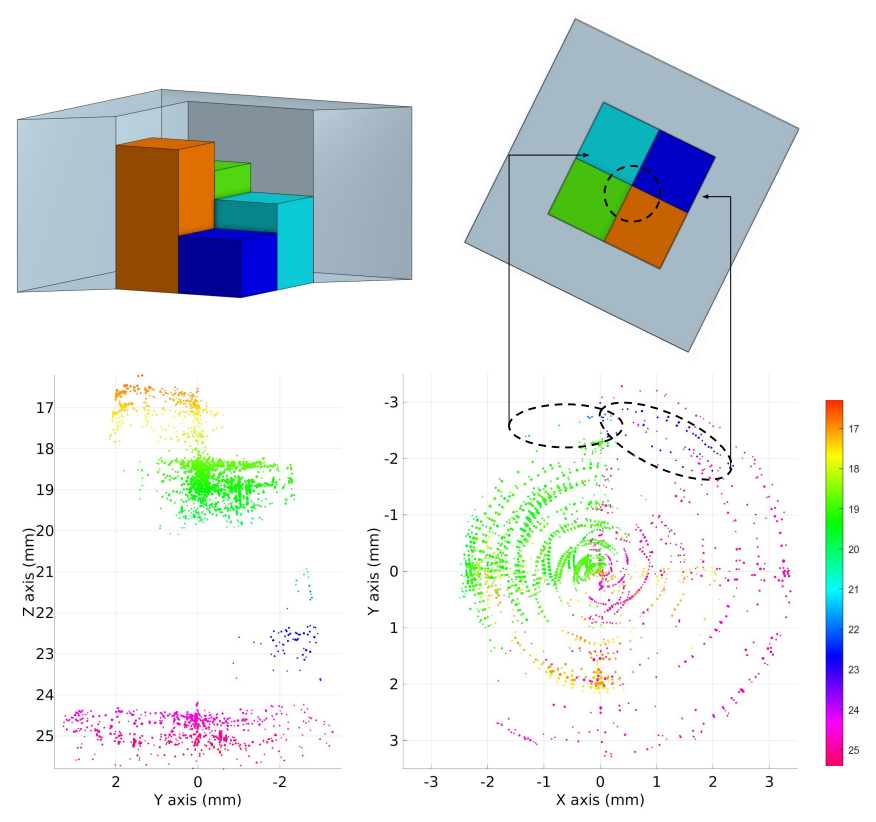

Fig. 8. C-scans of the stepped phantom. The colour of the points is encoded to the depth along the $\mathrm{Z}$ axis while the size of the points is the intensity of the signal recorded. Top Row - CAD renders of the stepped phantom with angled sectioned view (left) and top down view (right), each render is aligned to C-scan below it, the black dotted circle indicates the C-scans field of view. Each of the coloured levels is $5 \times 5 \mathrm{~mm}$ and the distance between each level is $2 \mathrm{~mm}$. Bottom Row - constructed C-scan shown from the side, YZ (left) and top-down, XY (right) black dotted ellipses indicate the areas on the C-scan that relate to the light and dark blue sections of the phantom.

static and scanning footprint of the instrument maintains the suitability for the targeted fetal procedures, as the procedures require low instrument diameters for introduction but then are performed within a cavity.

The mechanism presented in this paper utilises a rotating square shaft coupled to a sliding gear and a leadscrew and nut to rotate and translate the tube respectively. This approach allows the motors to be fixed in position at the proximal side of the mechanism. A design following this approach was previously presented in [25]. The core improvements to this mechanism are in the inclusion of linear rails to reduce the load on the nitinol tube during linear motion and the use of Oldham couplings for transmitting torque between the motor pack and carriage pack. Additionally, the overall size of the instrument has been reduced, the moving components have been enclosed in a housing and the power and data are all routed through a single multi core cable.

One of the main novelties in the end-effector design is the use of the torque coil between the curved nitinol tube and the OpUS sensor. This allowed continuous rotation of the curved nitinol tube while maintaining the axial orientation of the OpUS. Without the torque coil, a torsion would be applied to the fibre-optic sensor, influencing the signal and potentially result in structural damage to the OpUS sensor. Rotation junctions for fibres could have also been applied to this problem however multiple fibre junctions are complex and expensive mechanisms that would restrict the sensors used, in terms of the number of channels required. Whereas the torque coil approach only limits the sensor used in terms of diameter.
A possible source of uncertainty is elastic behaviour of the torque coil, which can introduce some errors into the position of the OpUS sensor relative to the nitinol tube.

A key aspect of scanning mechanisms is the repeatability of the motion [14], [17]. The repeatability of the mechanism is assessed using an EM tracker while in motion. The repeatability measured averaged $145 \mu \mathrm{m}$, with a maximum and minimum of $793 \mu \mathrm{m}$ and $17 \mu \mathrm{m}$ respectively. It should be noted that these values are past the validated accuracy of the EM tracking system, however these are just used as an indication for the repeatability of the mechanism and the values are not used to calibrate or generate the presented C-scans. The repeatability is generally higher in the middle sections of the linear joint limits which for the joint trajectories used while scanning would be when the sensor is moving at the highest velocities. These higher values could partially be attributed to the EM sensor tracking losing some stability at higher velocities, thus increasing the error of the measurements [26]. Additionally, the synchronisation process between the EM sensor data and joint state relies on the timestamps which at high velocities may introduce discrepancies.

The OpUS calibration allows for an approximation of the configuration of the mechanism. While the curvature of the nitinol tube can be measured, the position of the sensor relative to the torque coil, the position of the torque coil relative to the nitinol tube and the zero position of the nitinol tube are significantly harder to measure physically. These unknowns can be encompassed in the parameters of backlash or the deadzone in axial motion due to change of direction and the curvature or the path followed by the sensor when extended. The calibration procedure attempts to compensate for the unknowns with the parameters by imaging a known geometric object and assessing the reconstruction with respect to these parameters. The parameters were found via imaging a planar surface with a raised line through the centre. As shown in Fig. 4, the two parameters are reliant on different metrics, with the backlash using the distance between planes and the curvature using the width of the raised line. The parameters identified here using the line phantom were $53.5 \mathrm{~mm}$ and $1.49 \mathrm{~mm}$ for the curvature and backlash respectively. The width error and height error were $85.7 \mu \mathrm{m}$ and $76.1 \mu \mathrm{m}$ respectively. The curvature found is slightly increased compared to the value found using the EM tracking data, which likely is from the added stiffness of the OpUS fibres. The calibration procedure determines the physical parameters of the mechanism, however the calibration procedure used the same scan trajectory parameters and imaging medium. It is uncertain if these parameters are linked to the scan trajectory parameters and the imaging medium, or if the influence from changes in either would have a negligible effect on the images acquired.

Images of each phantom were constructed by combining the acquired A-scan and the pose of the sensor determined by the kinematics. This form of image is commonly referred to as a C-scan, differing from the images seen from conventional ultrasound transducers known as B-mode which require beamforming and a reconstruction step to form the images. This type of imaging is currently not possible with 
the instrumentation as B-mode reconstructions require high positioning accuracy of the transducer $(<50 \mu \mathrm{m})$ [27], [28]. $\mathrm{C}$-scans have a lower dynamic range than B-mode imaging, however the difference is minimised from the use of highly directional receivers. Whilst $\mathrm{C}$-scans do not provide the same level of contrast as B-mode imaging, porcine studies with OpUS have shown the information provided by $\mathrm{C}$-scans can yield clinically relevant information, and enable the identification of anatomical structures [4].

The calibrated scanning mechanism was then assessed using four phantoms of increasing complexity. The raised line and cross phantoms could be distinguished clearly from the constructed C-scans. The error in the height offset between the top and base surfaces, as compared to the measured value, was $10 \mu \mathrm{m}$ and $80 \mu \mathrm{m}$ for the line and cross, respectively. The points on the top level of the C-scan fit the measured geometric model of the line phantom $98.36 \%$ and all of the points fit within. For the cross phantom, $95.9 \%$ of the points were within the measured geometric model and all of the points were fit within a width of $3.60 \mathrm{~mm}$, and the points outside of the measured width can be identified visually as close to the corners of the cross.

For the stepped phantom, whilst the levels of the phantom can be distinguished readily, in the bottom-left section of Fig. 8, the lateral information of the C-scan (bottom-right of Fig. 8) is difficult to interpret. There are a number of possible reasons for this. One is a misalignment of the phantom under the scanning mechanism, attributable to the manual alignment between the scanning mechanism and the phantom (which is assessed from visually inspecting the live A-scan over a single scan iteration). This would correspond to the views seen if the centre of the phantom was offset from the central axis of the instrument as indicated on the CAD render in Fig. 8. Increasing the offset along the $\mathrm{Z}$ axis between the phantom and scanning mechanism would increase the field of view and potentially show a larger portion of the phantom. However the imaging depth is limited (ca. $25 \mathrm{~mm}$ ) and this could result in the phantom not being fully imaged.

The scanning mechanism operates in a continuous manner acquiring scan data constantly, additionally the Cartesian path of the probe varies on each rotation (as seen in Fig. 7).

This allows the scan density to be determined by the acquisition time for a given set of scanning trajectory parameters. The scanning parameters can also influence the scan density through the rotation speed and linear velocity and acceleration profiles; the range of motion of the linear joint and the curvature of the nitinol tube then determine the field of view. Scanning trajectories are generated through a consistent and repeatable trajectory in joint space; this does not translate into a uniform Cartesian scan but instead enables the scan density to be linked to the acquisition time. The scans presented in this paper were formed over $10 \mathrm{~s}$ and use the information from approximately 1000 A-scans. Optimum scan parameters will vary depending on the procedure and the intention (navigation, guidance, or diagnosis), the focus of this paper has been on the development of the instrument rather than optimising for a single procedure.

The sides of the C-scans exhibit distortions due to inac- curacies in the modelled motion of the OpUS sensor. The distortions are approximately $250-500 \mu \mathrm{m}$, highlighted in Fig. 6. This appears to be an error in the C-scan construction process likely due to uncompensated axial motion of the sensor within the torque coil leading to inaccuracies in the calculated pose of the OpUS sensor. The sensor is currently not constrained within the torque coil at the distal end, therefore, it can still move within the coil. An additional consideration is that the current kinematic model assumes there is no deformation of the steel instrument shaft, and no torsion in the nitinol tube. Both of these factors may introduce torsional "wind-up" when the sensor is at the lower limits of the linear joint, which is then released when the sensor is at the upper limits of the linear joint. This would cause a difference in the tube rotation depending on the linear position.

\section{CONCLUSION}

This paper has presented the design, fabrication and initial characterisation of a concentric tube based scanning mechanism. The instrument follows a modular design utilising Oldham couples with two pins either side to align and transmit torque between the carriage section and the motor section. The carriage is supported by linear rails either side of the nitinol tube to reduce load on the tube. An OpUS probe was integrated into the instrument through the use of a torque coil, and this allowed the nitinol tube to rotate continuously without passing the torsion onto the sensor. The overall instrument diameter was $2.45 \mathrm{~mm}$, while the maximum diameter while scanning was less than $6 \mathrm{~mm}$. Assembly of the scanning data into a $\mathrm{C}$-scan was enabled through a calibration procedure that relied on a small phantom incorporating a raised line in running through the middle, and this allowed the kinematic parameters of the instrument to be determined numerically via a parameter sweep. The quality of reconstructed C-scans were then assessed using four phantoms, each with increasing geometric complexity. The $\mathrm{C}$-scans for the line and cross phantoms provided clear and accurate scans: here, the planarity of the surfaces averaged $0.27 \mathrm{~mm}$. However for the stepped phantom, while the axial information displays clear levels, the lateral detail remains unclear. These results illustrate the potential of robot-assisted scanning for the acquisition of Cscans with OpUS sensors. One of the advantages of this approach is the scanning aperture and scan density can be adjusted through the trajectory or tube design whilst maintaining a small footprint for introduction. Future work will focus on improving the $\mathrm{C}$-scans through attempting to model the OpUS probe motion more accurately and optimise the tube parameters and scanning trajectory for the desired field of view and scan density. Additional experimental work is also needed to understand how this type of device would function within a more realistic environment with tissues, physiological motion and other clinically relevant conditions.

\section{ACKNOWLEDGMENT}

The authors would like to thank Dr. Efthymios Maneas for his help in setting up the experiments and valuable discussions. 


\section{REFERENCES}

[1] R. J. Colchester, E. Z. Zhang, C. A. Mosse, P. C. Beard, I. Papakonstantinou, and A. E. Desjardins, "Broadband miniature optical ultrasound probe for high resolution vascular tissue imaging," Biomed. Opt. Express, vol. 6, no. 4, p. 1502, apr 2015. [Online]. Available: https://www.osapublishing.org/abstract.cfm?URI=boe-6-4-1502

[2] S. Noimark, R. J. Colchester, B. J. Blackburn, E. Z. Zhang, E. J. Alles, S. Ourselin, P. C. Beard, I. Papakonstantinou, I. P. Parkin, and A. E. Desjardins, "Carbon-Nanotube-PDMS Composite Coatings on Optical Fibers for All-Optical Ultrasound Imaging," Adv. Funct. Mater, vol. 26, no. 46, pp. 8390-8396, dec 2016. [Online]. Available: http://doi.wiley.com/10.1002/adfm.201601337

[3] R. J. Colchester, C. Little, G. Dwyer, S. Noimark, E. J. Alles, E. Z. Zhang, C. D. Loder, I. P. Parkin, I. Papakonstantinou, P. C. Beard, M. C. Finlay, R. D. Rakhit, and A. E. Desjardins, "All-Optical Rotational Ultrasound Imaging," Sci. Rep., vol. 9, no. 1, p. 5576, dec 2019. [Online]. Available: http://www.nature.com/articles/s41598-019-41970-z

[4] M. C. Finlay, C. A. Mosse, R. J. Colchester, S. Noimark, E. Z. Zhang, S. Ourselin, P. C. Beard, R. J. Schilling, I. P. Parkin, I. Papakonstantinou, and A. E. Desjardins, "Through-needle all-optical ultrasound imaging in vivo: a preclinical swine study," Light Sci. Appl., vol. 6, no. 12, p. e17103, dec 2017. [Online]. Available: http://www.nature.com/doifinder/10.1038/lsa.2017.103

[5] C. Gruijthuijsen, R. Colchester, A. Devreker, A. Javaux, E. Maneas, S. Noimark, W. Xia, D. Stoyanov, D. Reynaerts, J. Deprest, S. Ourselin, A. Desjardins, T. Vercauteren, and E. V. Poorten, "Haptic Guidance Based on All-Optical Ultrasound Distance Sensing for Safer Minimally Invasive Fetal Surgery," $J$. Med. Robot. Res., p. 1841001, mar 2018. [Online]. Available: https://www.worldscientific.com/doi/abs/10.1142/S2424905X18410015

[6] J. Smits, M. Ourak, A. Gijbels, L. Esteveny, G. Borghesan, L. Schoevaerdts, K. Willekens, P. Stalmans, E. Lankenau, H. SchulzHildebrandt, G. Huttmann, D. Reynaerts, and E. Vander Poorten, "Development and Experimental Validation of a Combined FBG Force and OCT Distance Sensing Needle for Robot-Assisted Retinal Vein Cannulation," in 2018 IEEE Int. Conf. Robot. Autom. IEEE, may 2018, pp. 129-134. [Online]. Available: https://ieeexplore.ieee.org/document/8460983/

[7] W. Xia, S. J. West, M. C. Finlay, J.-m. Mari, S. Ourselin, A. L. David, and A. E. Desjardins, "Looking beyond the imaging plane: 3D needle tracking with a linear array ultrasound probe," Sci. Rep., vol. 7, no. 1, p. 3674, dec 2017. [Online]. Available: http://www.nature.com/articles/s41598-017-03886-4

[8] E. J. Alles, S. Noimark, E. Maneas, E. Z. Zhang, I. P. Parkin, P. C. Beard, and A. E. Desjardins, "Video-rate all-optical ultrasound imaging," Biomed. Opt. Express, vol. 9, no. 8, pp. 3481-3494, Aug 2018. [Online]. Available: http://www.osapublishing.org/boe/abstract.cfm?URI=boe-98-3481

[9] D. T. Yeh, O. Oralkan, I. O. Wygant, M. O'Donnell, and B. T. KhuriYakub, "3-d ultrasound imaging using a forward-looking CMUT ring array for intravascular/intracardiac applications," IEEE Transactions on Ultrasonics, Ferroelectrics, and Frequency Control, vol. 53, no. 6, pp. 1202-1211, 2006

[10] C. Tekes, T. Xu, T. M. Carpenter, S. Bette, U. Schnakenberg, D. Cowell, S. Freear, O. Kocaturk, R. J. Lederman, and F. L. Degertekin, "Real-time imaging system using a 12-mhz forward-looking catheter with single chip cmut-on-cmos array," in 2015 IEEE International Ultrasonics Symposium (IUS), 2015, pp. 1-4.

[11] G. Dwyer, R. J. Colchester, E. J. Alles, E. Maneas, S. Ourselin, T. Vercauteren, J. Deprest, E. V. Poorten, P. D. Coppi, A. E. Desjardins, D. Stoyanov, E. Vander Poorten, P. De Coppi, A. E. Desjardins, and D. Stoyanov, "Robotic Control of a Multi-Modal Rigid Endoscope Combining Optical Imaging with All-Optical Ultrasound," in 2019 Int. Conf. Robot. Autom. IEEE, may 2019, pp. 3882-3888. [Online]. Available: https://ieeexplore.ieee.org/document/8794289/

[12] R. J. Colchester, E. J. Alles, G. Dwyer, E. Maneas, D. Stoyanov, and A. E. Desjardins, "Large area all-optical ultrasound imaging using robotic control," in Opto-Acoustic Methods and Applications in Biophotonics IV, V. Ntziachristos and R. Zemp, Eds., vol. 11077, International Society for Optics and Photonics. SPIE, 2019, pp. 107 - 111. [Online]. Available: https://doi.org/10.1117/12.2525379

[13] P. Giataganas, M. Hughes, C. J. Payne, P. Wisanuvej, B. Temelkuran, and G.-Z. Yang, "Intraoperative Robotic-Assisted Large-Area HighSpeed Microscopic Imaging and Intervention," IEEE Trans. Biomed. Eng., vol. 66, no. 1, pp. 208-216, jan 2019. [Online]. Available: https://ieeexplore.ieee.org/document/8359328/
[14] M. S. Erden, B. Rosa, N. Boularot, B. Gayet, G. Morel, and J. Szewczyk, "Conic-spiraleur: A miniature distal scanner for confocal microlaparoscope," IEEE/ASME Trans. Mechatronics, vol. 19, no. 6, pp. 1786-1798, 2014.

[15] H. Wang, Z. Ping, Y. Fan, H. Kang, and S. Zuo, "A novel surfacescanning device for intraoperative tumor identification and therapy," IEEE Access, vol. 7, pp. 96392-96403, 2019.

[16] G. Dwyer, P. Giataganas, P. Pratt, M. Hughes, and G.-Z. Yang, "A miniaturised robotic probe for real-time intraoperative fusion of ultrasound and endomicroscopy," in 2015 IEEE Int. Conf. Robot. Autom. IEEE, may 2015, pp. 1196-1201. [Online]. Available: http://ieeexplore.ieee.org/document/7139343/

[17] E. J. Seibel, Q. Y. J. Smithwick, C. M. Brown, and P. G. Reinhall, "Single fiber flexible endoscope: general design for small size, high resolution, and wide field of view," SPIE Proc., vol. 4158, no. 2001, pp. 29-39, Jan 2001. [Online]. Available: http://proceedings.spiedigitallibrary.org/proceeding. .

[18] Y. Li, R. Lin, C. Liu, J. Chen, H. Liu, R. Zheng, X. Gong, and L. Song, "In vivo photoacoustic/ultrasonic dual-modality endoscopy with a miniaturized full field-of-view catheter," Journal of Biophotonics, vol. 11, no. 10, p. e201800034, 2018. [Online]. Available: https://onlinelibrary.wiley.com/doi/abs/10.1002/jbio.201800034

[19] J. Burgner, P. J. Swaney, R. a. Lathrop, K. D. Weaver, and R. J. Webster, "Debulking from within: A robotic steerable cannula for intracerebral hemorrhage evacuation," IEEE Trans. Biomed. Eng., vol. 60, no. 9, pp. 2567-2575, sep 2013. [Online]. Available: http://ieeexplore.ieee.org/lpdocs/epic03/wrapper.htm?arnumber=6510505

[20] J. A. Guggenheim, J. Li, T. J. Allen, R. J. Colchester, S. Noimark, O. Ogunlade, I. P. Parkin, I. Papakonstantinou, A. E. Desjardins, E. Z. Zhang, and P. C. Beard, "Ultrasensitive plano-concave optical microresonators for ultrasound sensing," Nat. Photonics, vol. 11, no. 11, pp. 714-719, nov 2017. [Online]. Available: http://www.nature.com/articles/s41566-017-0027-x

[21] E. Z. Zhang and P. C. Beard, "Characteristics of optimized fibre-optic ultrasound receivers for minimally invasive photoacoustic detection," in Photons Plus Ultrasound: Imaging and Sensing 2015, A. A. Oraevsky and L. V. Wang, Eds., vol. 9323, International Society for Optics and Photonics. SPIE, 2015, pp. 151 - 159. [Online]. Available: https://doi.org/10.1117/12.2081904

[22] J. H. Ma, S. Sefati, R. H. Taylor, and M. Armand, "An active steering hand-held robotic system for minimally invasive orthopaedic surgery using a continuum manipulator," IEEE Robotics and Automation Letters, vol. 6, no. 2, pp. 1622-1629, 2021.

[23] P. Dupont, J. Lock, B. Itkowitz, and E. Butler, "Design and control of concentric-tube robots," IEEE Trans. Robot., vol. 26, no. 2, pp. 209-225, apr 2010. [Online]. Available: http://ieeexplore.ieee.org/xpls/abs_all.jsp?arnumber $=5371822$

[24] R. J. Colchester, E. J. Alles, and A. E. Desjardins, "A directional fibre optic ultrasound transmitter based on a reduced graphene oxide and polydimethylsiloxane composite," Appl. Phys. Lett., vol. 114, no. 11, p. 113505, mar 2019. [Online]. Available: http://aip.scitation.org/doi/10.1063/1.5089750

[25] G. Dwyer, F. Chadebecq, M. T. Amo, C. Bergeles, E. Maneas, V. Pawar, E. V. Poorten, J. Deprest, S. Ourselin, P. De Coppi, T. Vercauteren, and D. Stoyanov, "A Continuum Robot and Control Interface for Surgical Assist in Fetoscopic Interventions," IEEE Robot. Autom. Lett., vol. 2, no. 3, pp. 1656-1663, jul 2017. [Online]. Available: http://ieeexplore.ieee.org/document/7874155/

[26] E. Bonmati, Y. Hu, K. Gurusamy, B. Davidson, S. P. Pereira, M. J. Clarkson, and D. C. Barratt, "Assessment of electromagnetic tracking accuracy for endoscopic ultrasound," in Lect. Notes Comput. Sci. (including Subser. Lect. Notes Artif. Intell. Lect. Notes Bioinformatics), vol. 10170 LNCS. Springer, 2017, pp. 36-47. [Online]. Available: http://link.springer.com/chapter/10.1007/978-3-319-54057-3_4

[27] E. J. Alles, S. Noimark, E. Zhang, P. C. Beard, and A. E. Desjardins, "Pencil beam all-optical ultrasound imaging," Biomed. Opt. Express, vol. 7, no. 9, p. 3696, sep 2016. [Online]. Available: https://www.osapublishing.org/abstract.cfm?URI=boe-7-9-3696

[28] E. J. Alles and A. E. Desjardins, "Source density apodization: Image artifact suppression through source pitch nonuniformity," IEEE Transactions on Ultrasonics, Ferroelectrics, and Frequency Control, vol. 67, no. 3, pp. 497-504, 2020. 